\title{
Histological and Biochemical Evidence for an intravital Stab Wound on Ötzi's Right Hand
}

\author{
Andreas G. Nerlich', Beatrice E. Bachmeier²,Albert Zink', Eduard Egarter-Vig|3 \\ I Dept. of Pathology,Academic Hospital Munich-Bogenhausen, Englschalkingerstr. 77, D-8I 925 München, Germany \\ 2 Dept. of Clin. Chemistry Clin. Biochemistry, Ludwig-Maximilians-University Munich, \\ Nussbaumstr. 20, D-80336 München, Germany, \\ 3 Dept. of Pathology, Province Hospital Bozen/Bolzano, Lorenz-Böhler-Str. 5, I-39100 Bozen/Bolzano, Italy
}

KEY WORDS: Frozen mummy, Tyrolean Iceman, trauma, histology, blood analysis
Abstract
On careful re-examination of the mummy a small irregularly shaped $3.7 \mathrm{~cm}$ long, deep, dark brown coloured stab wound was detected on Ötzi's right hand extending from the palmar hand to the lateral back of the hand. Since it was unclear whether this wound had happened during lifetime or whether it was a postmortal artefact, we performed a histological analysis including histochemical staining for haemosiderin deposits (on Prussian blue stains). The fat tissue revealed cord-like inclusions of an amorphous, autofluorescing material with isolated focal haemosiderin pigment droplets in Prussian blue staining without intact erythrocytes. This amorphous material was then isolated by laser based microdissection, dissolved and tested for the presence of haemoglobin. A Guaiac-based test confirmed the presence of a blood clot. Our investigations indicate that the Iceman suffered from a vital stab wound of the right hand. Typical bleeding into the subcutaneous fat tissue and the initiation of transformation of the blood clot into haemosiderin could be demonstrated. Since forensic medical practise suggests the occurence of haemosiderin containing macrophages in skin wounds between 3 to 8 days after affliction, we strongly suggest that the injury happened a few days before his death. At present, we do not know if this skin wound may have happened simultaneously or shortly after Ötzi was hit by the arrow, but if so this would suggest that he survived the attack for a few days.

\section{Introduction}

Recent publications have assumed that no further information will come from the c. 5300 year old mummy of the Tyrolean Iceman, commonly known as „Ötzi”(Sharp, 2002). In contrast, the present report describes histological and biochemical evidence that Ötzi suffered from a vital wound on his right hand which he may have suffered a few days before his death. This finding sheds some important light on the last few days in Ötzi's life. It also suggests that a careful investigation may provide new information about the iceman's final days.

When the iceman was revealed in September 1991 due to the receding glacier of Val Senales (South Tyrol, north Italy), the mummified body suffered from several skeletal and soft tissue injuries due to the manipulations when the corpse was removed from the ice. These had to be added to several injuries that may have happened after his death when the mummy became encased in the glacier. A recent survey summarizes numerous fractures of various bones and subluxations of costovertebral joints as postmortal injuries (Murphy et al., 2003). Furthermore, the left pelvis was so severely damaged that the left ischium and other bones, such as part of the sacrum and soft tissue structures of that location were completely absent (Murphy et al., 2003).

Apart from these clear artefacts, a recent re-examination of thoracic CT scans provided evidence for a presumably intravital arrow injury (Gostner and Egarter-Vigl, 2002; Holden, 2003). Concomitantly, a small skin wound was detected on the iceman's back which was identified as the entry point of the arrow. This finding strongly suggested that Ötzi had died from a lethal assault (Gostner and Egarter-Vigl, 2002).

\section{Methods and observations}

Very recently, one of our study group (E.E.V.) carefully reexamined the mummy and detected a small irregularly shaped stab wound on Ötzi's right hand. This presented as an approx. $3.7 \mathrm{~cm}$ long defect of the palmar hand extending to the lateral back of the hand (Fig. 1). The wound margins are wavy, dark brown coloured and the defect seems to extend deeply into the soft tissue reaching the metacarpal 

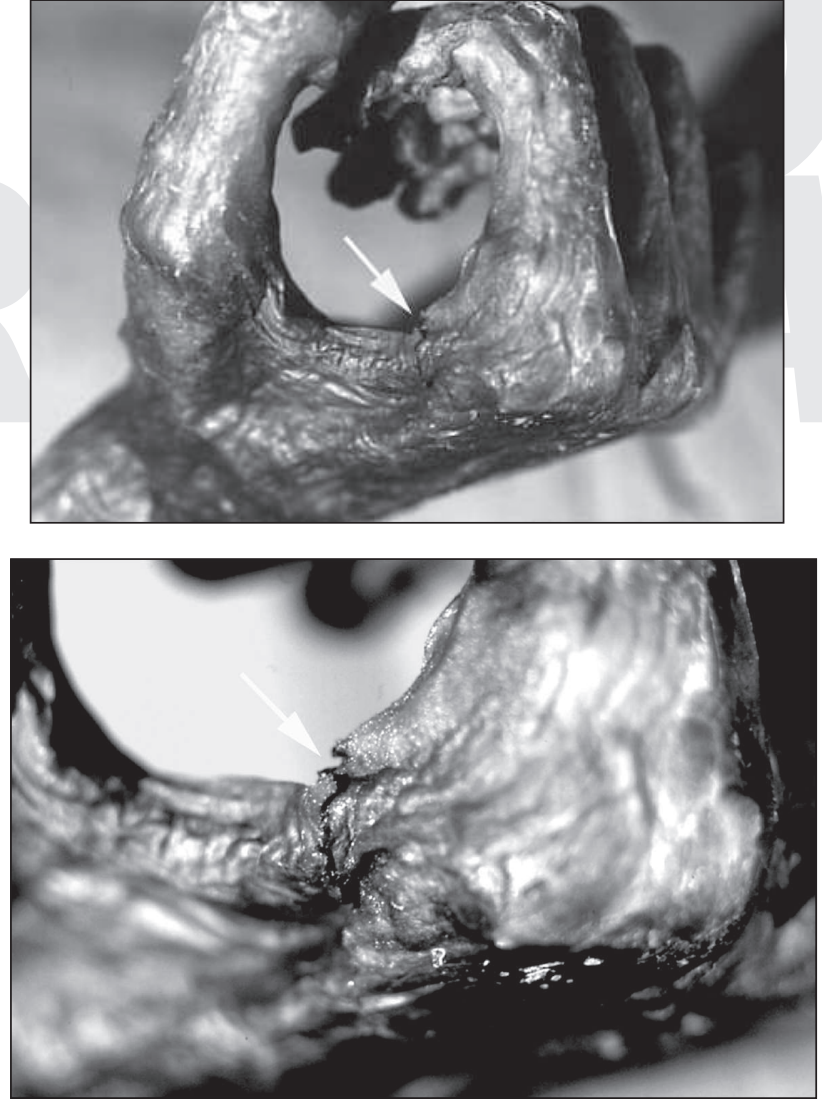

Fig. 1 - Macroscopic view of the stab wound on Ötzi's right hand. (A) The irregularly shaped, wavy defect (arrow) extends from the palm laterally. (B) On closer view the undulated margins of the wound and the brown coloration are seen (arrow).

bone which shows a small osseous defect at the wound site. Macromorphologically it was unclear whether this wound had happened during lifetime or whether it was a postmortal artefact. We therefore removed a small tissue sample from that site. This was carefully rehydrated and embedded into paraffin wax for histological analysis as previously performed on mummy tissue (Nerlich et al., 1995) including histochemical staining for haemo-siderin deposits by using Prussian blue stains.

The resulting morphology provided very well preserved and normally appearing dermal collagen fibers and subcutaneous fat with slight adipocere formation which is well in concordance with the previous histological evidence for postmortal slight diagenetic alterations of the mummy's tissue (Hess et al., 1998). The fat tissue additionally revealed cord-like inclusions of an amorphous, strongly red autofluorescing material (Fig. 2) that contained isolated focal haemosiderin pigment droplets in Prussian blue staining (Fig. 3). No cell nuclei or typical erythrocytes were seen. A further analysis by use of high resolution scanning force microscopy similarly demonstrated no intact erythrocytes. However, other tissue constituents, such as collagen fibrils were adequately well preserved providing typical cross-banding pattern of the collagen fibers and showing no abnormality in the collagenous network. In order to confirm these morphological observations, we subsequently used a section to test for the presence of haemoglobin using both a haemoglobin antibody test and a

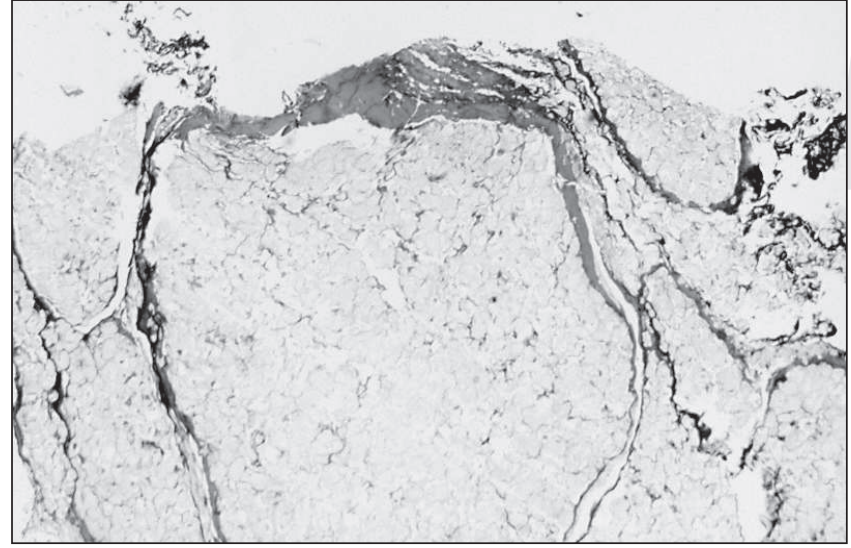

Fig. 2 - Histological features of the wound area showing an amorphous material between collagen fibers and fat tissue (van Gieson's connective tissue stain, original $\times 100$ ).

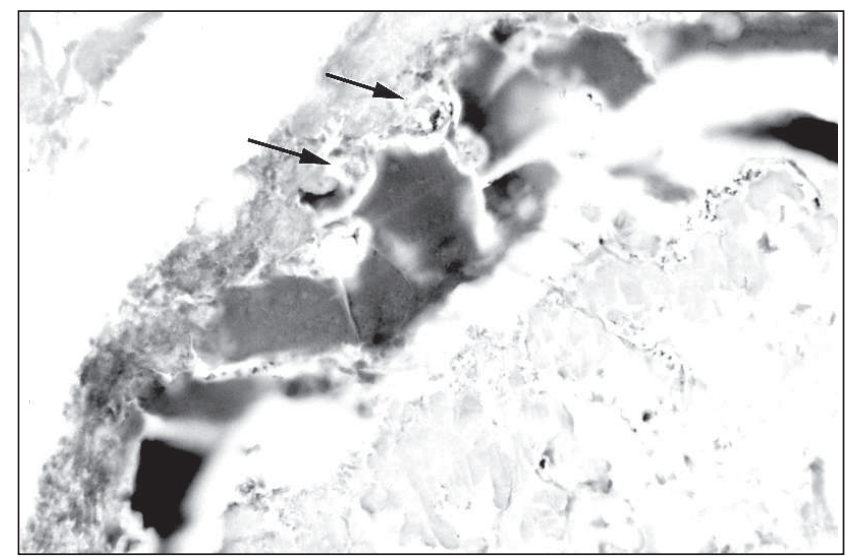

Fig. 3 - Note the presence of small haemosiderin deposits in this amorphous material suggesting remnants of blood phagocytosing macrophages (Prussian blue, original x 450).

routine Guaiac-based test for pseudoperoxidase reaction of haemoglobin (Ahlquist, 1992). Both tests initially did not provide a positive result, most probably due to the small amount of clotted blood and an intimate mixture with other tissue components. Therefore, this experiment was repeated and the tissue region of interest was selected by using laser based microdissection (Thalhammer et al., 2003) to selectively isolate the amorphous material. In order to enrich the material, this was pooled from several sections, dissolved mechanically and intensely in a minute amount of phosphate-buffered saline and then again tested for the presence of haemoglobin. While the antibody test failed (probably due to a loss of preserved antigens), the Guaiacbased test provided a faintly, but clearly positive result, thereby confirming the presence of a blood clot.

\section{Discussion}

Our investigations indicate that the Iceman suffered from a vital stab wound of the right hand. Typical bleeding into the subcutaneous fat tissue and the initiation of transformation of the blood clot into haemosiderin (despite no longer identifiable macrophages) could be demonstrated by a twofold approach: both the histochemical analysis of the 
subcutaneous skin revealed typical positive heamosiderin droplets in the obvious residues of macrophages and the biochemical testing for haemoglobin in a routine test revealed a positive result. Most interestingly, the bleeding substance was so little that only the pooling of selected areas of the region of interest by use of a laser beam microdissection unit provided a positive result.

The presence of haemosiderin is a clear proof for an intravital reaction since the histochemical stain used in the examination specifically and exclusively reacts only with trivalent iron, but not with the divalent form present in haemoglobin (Böck, 1989). In summary, this study provides a dual evidence for the bleeding as shown by the presence of haemoglobin (biochemically) and haemosiderin (by histochemistry).

Since forensic medical practise suggests the occurence of haemosiderin containing macrophages in skin wounds between 3 to 8 days after affliction (Betz et al., 1996), we strongly suggest that the injury happened a few days before the iceman's death. At present, we do not know if this skin wound may have happened simultaneously or shortly after Ötzi was hit by the arrow, but if so this would suggest that he survived the attack for a few days. We suggest that the ongoing careful examination of the Iceman will provide further significant information on his life and possibly his death.

\section{Literature Cited}

Ahlquist DA, Occult blood screening: obstacles and effectiveness. Cancer 1992; 70 (Suppl.): I 259- 1265.

Betz P, Eisenmenger W, Morphometrical analysis of hemosiderin deposits in relation to wound age. Int J Legal Med 1996; 108:262-4.

Böck P, Mikroskopische Technik. Urban and Fischer, Munich 1989, Pp.399-400.

Gostner P, Egarter-Vigl E, Report of radiological-forensic findings on the iceman. J Archaeol Sci 2002; 29: 323-326.

Hess MW, Klima G, Pfaller K, Künzel KH, Gaber O, Histological investigations on the Tyrolean Iceman. Am J Phys Anthropol 1998; 106: 521-532.

Holden C, Ötzi death riddle solved. Science 200I; 293: 795.

Murphy WA Jr, zur Nedden D, Gostner P, Knapp R, Recheis W, Seidler H,The Iceman: Discovery and imaging. Radiology 2003; 226: 614-629.

Nerlich A, Parsche F, Wiest I, Schramel P, Löhrs U, Extensive pulmonary haemorrhage in an Egyptian mummy. Virchows Arch 1995; 427: 423-429.

Sharp D.Time to leave Ötzi alone? Lancet 2002; 360: I530.

Thalhammer S, Lahr G, Clement-Sengewald A, HecklWM,

Burgemeister R, Schütze K. Laser microtools in cell biology and molecular medicine. Laser Physics 2003; I 3: 68I-692. 